\title{
LAS POLÍTICAS PÚBLICAS DEL FOMENTO AL CRECIMIENTO EN CHILE. ANÁLISIS DEL PRIMER AÑO DEL GOBIERNO DE Sebastián Piñera
}

Francisco Agüero (faguero@derecho.uchile.cl)

Pablo Rivadeneira (privadeneira@gmail.com)

Centro de Regulación y Competencia

Universidad de Chile.

Este artículo evalúa los resultados de la incorporación del Estatuto PYME en el sistema de análisis regulatorio, que surge como una novedosa iniciativa para aumentar la eficiencia de las políticas publicadas orientadas a las pequeñas y medianas empresas durante el periodo 2010-2011. Los resultados demuestran queexiste un débil marco regulatorio para las empresas de menor tamaño, como consecuencia de la falta de habido apoyo efectivo desde cada uno de los ministerios involucrados, con excepción del Ministerio de Economía.

Palabras Clave: economía, regulación, pequeñas y medianas empresas, Chile.

\section{THE PUBLIC POLICY OF GROWTH PROMOTION IN CHILE. AN ANALYSIS OF SEBASTIÁN PIÑERA'S FIRST YEAR IN GOVERNMENT}

This paper analyses the effects of the introduction of the "Estatuto PYME" as a novel initiative aimed at increasing the efficiency of public policies in small or medium-sized enterprises (PYME) during 2010-2011. A review of the data shows a weak regulatory framework for PYMEs, due in large part to minimal effective support from ministries, with the exception of the Ministry of the Economy.

Keywords: economy, regulation, small and medium sized enterprises, Chile. 


\section{Introducción ${ }^{1}$}

Hace dos décadas Chile era considerado un país pobre; hoy, una de las economías en vías de desarrollo más promisorias en su región. Tras la instauración de un modelo de mercado abierto, a principios de la década de 1980, y luego de profundos cambios macroeconómicos que consolidaron y potenciaron el modelo (entre 1990 y 2000), Chile es hoy un país con posibilidades reales de alcanzar el desarrollo económico dentro de la próxima década. El crecimiento chileno desde mediados de los ochenta se debe en parte a la continuidad, dada por los gobiernos a diversas políticas macroeconómicas, supervisadas por un Banco Central autónomo. Entre estas políticas destaca la fijación y cumplimiento de metas claras en materia inflacionaria y la instauración de un régimen de tipo de cambio flotante. A esto se debe agregar la consolidación de reformas tendientes a abrir la economía al comercio exterior, promoción de los sectores financiero e industrial, implementación de una nueva institucionalidad laboral y consolidación de un exitoso sistema de pensiones que ha incrementado la profundidad del sector financiero local.

Un rol importante en este desarrollo ha jugado la aplicación de una política fiscal sobria y regulada, la cual ha permitido compatibilizar un crecimiento sostenido en inversiones sociales con un ambiente fiscalmente ordenado. La estabilidad democrática, consolidación institucional y respeto por el imperio del derecho (rule of law), han permitido al país situarse a la vanguardia en los indicadores de desarrollo económico de la región e ingresar a la OCDE a fines de 2009.

Chile es una de las economías de América Latina que más rápidamente ha crecido. Desde 1986 a 1997 creció a una tasa promedio de 7,6\% anual, para descender a un 3,3\% anual entre 1998 y 2009 (ver gráfico 1). En las últimas décadas, el ingreso per cápita de los chilenos se duplicó en valores reales y las brechas de nuestros indicadores económicos respecto a los de los países desarrollados disminuyeron notablemente. Por ejemplo, respecto al de EE.UU, nuestro ingreso per cápita PPP se incrementó de $18 \%$, en 1986, a 31\% en 2007, superando con dicho aumento a todos los demás países latinoamericanos, salvo México (OCDE, 2011:54). En todo caso, debemos reconocer que gran parte de esta disminución en la brecha de nuestro ingreso per cápita proviene del período 1986 - 1997, ya que con posterioridad a dicho año, disminuyó abruptamente nuestra velocidad de crecimiento respecto al resto de las economías del mundo.

Este trabajo no podría haberse llevado a cabo sin la valiosa colaboración de los investigadores del Centro de Regulación y Competencia (RegCom) Daniela Fuenzalida, Consuelo Fernández, Daniela Navarro y Julio Arancibia. 


\section{Gráfico 1}

Crecimiento económico $1986-1997$ vs. 1998-2009

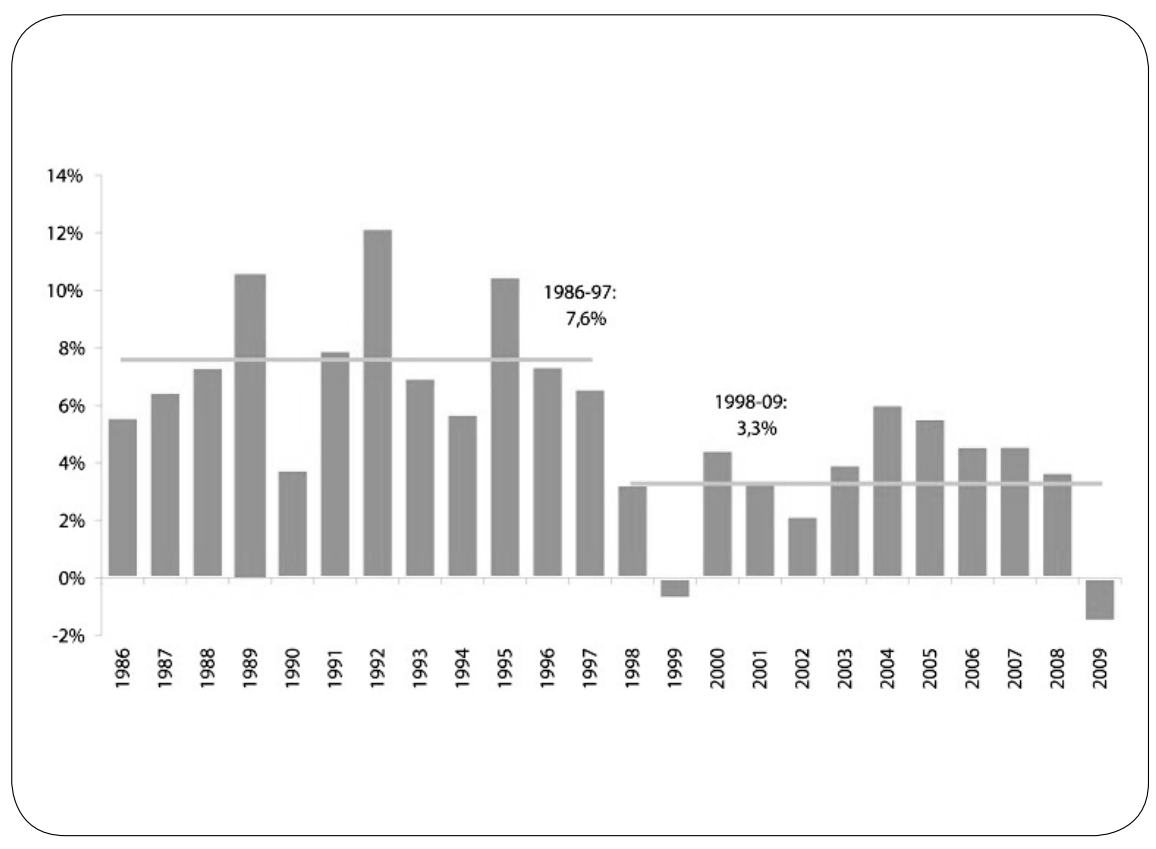

Fuente: Banco Central de Chile

Uno de los grandes desafios pendientes es disminuir la desigualdad del ingreso. El coeficiente de Gini de nuestro país alcanza hoy niveles de 0,5, lo cual nos sitúa como el país más desigual de la OCDE (tal como se señala en OCDE, 2011: 105). Entre 1990 y 2003, la distribución del ingreso autónomo de los hogares ${ }^{2}$ se mantuvo prácticamente constante. Recién en 2006 se registró una mejora sustancial en la distribución del ingreso: los deciles 2 a 9 aumentaron su participación, mientras que el decil 19 disminuyó de 41,5\% en 2003 a 38,6\% en 2006. En parte explicado por la crisis económica del año 2008, los datos sobre ingreso autónomo de 2009 revierten la anterior tendencia, pues grafican cómo entre el año 2006 y el 2009, sólo el decil 10 aumentó su participación, mientras los otros deciles disminuyeron, volviendo a sus niveles de 2003 (OCDE, 2011: 105).

Durante el período de alto crecimiento, comprendido entre 1986 a 1998, aumentaron considerablemente las exportaciones de recursos naturales, en especial, cobre, además

2 El ingreso autónomo del hogar se compone del ingreso que la familia genera con sus propios medios (ingreso por trabajo, rentas, intereses, pensiones, etc.), más otras transferencias que la familia recibe (por ejemplo, donaciones y aportes de familiares que no comparten el mismo hogar). 
de productos agrícolas, forestales, salmones, alimentos y vino (Bitrán y González, 2010: 6). Algunos datos demuestran lo anterior: en el periodo 1990-1995, las exportaciones de cobre crecieron en promedio 6,5\% y, entre 1996-2001, un 11,4\%. Las exportaciones no-cobre en esos periodos crecieron $11,2 \%$ y $7 \%$ respectivamente (Ffrench-Davis, 2002). No obstante, desde finales de los años 90 nuestra economía se ha visto afectada por una serie de crisis externas que han frenado considerablemente la anterior capacidad de crecimiento. En 1998 sufrimos los embates de la crisis asiática, la cual demostró la vulnerabilidad de nuestro modelo de país exportador. Ilustrativa es la caída sufrida a partir de dicho año por la inversión en capital fijo: de crecer a tasas promedio de promedio 13,5\% anual en 1986-1997, disminuyó a tasas de tan sólo 4,6\% entre 19982009 (Ministerio de Hacienda, 2010: 43).

\section{Gráfico 2}

Crecimiento de la productividad en Chile $1986-1997$ vs. $1998-2009$

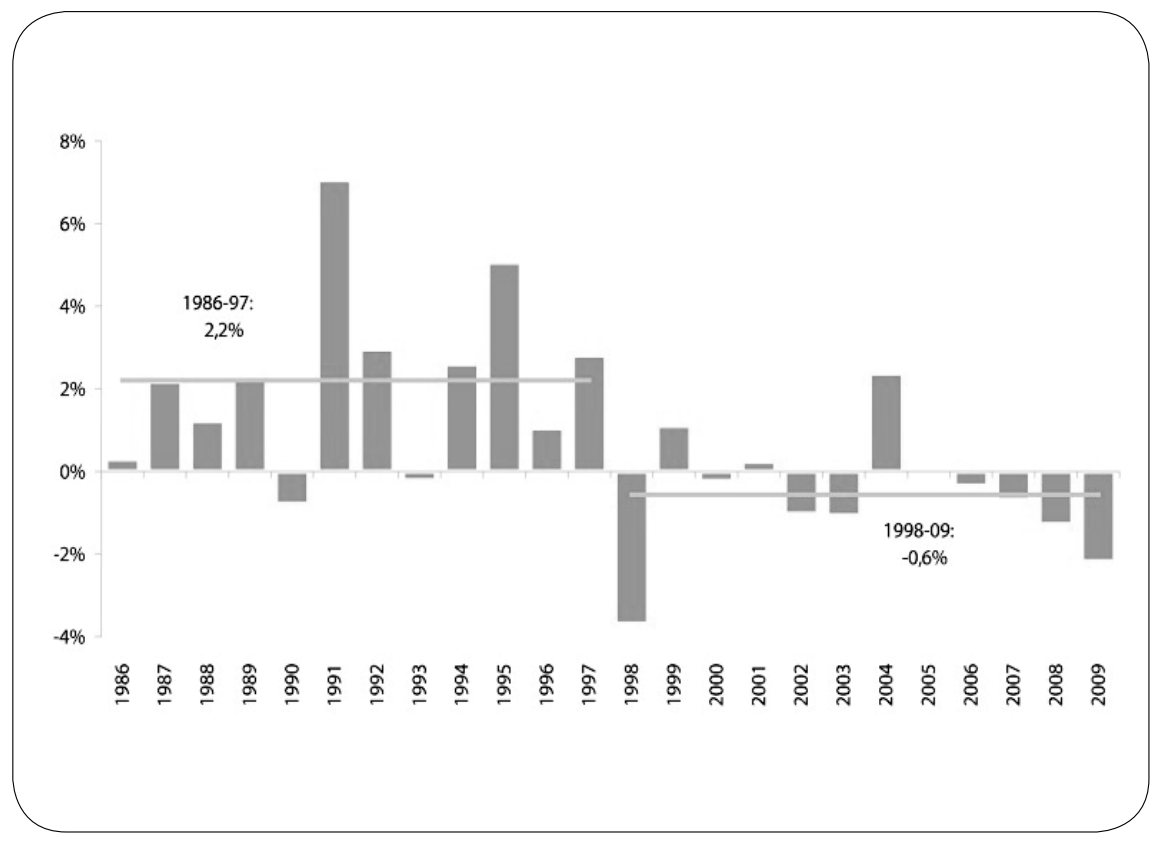

Fuente: Ministerio de Hacienda, INE y Banco Central de Chile

Chile tuvo también que lidiar con la crisis financiera Argentina en 2001 y con los posteriores recortes de gas natural. El efecto de estos recortes fue acentuado por el aumento en los precios mundiales del petróleo durante la pasada década, demostrándose la dependencia y falta de diversificación de nuestra matriz 
energética $^{3}$. La interrupción del abastecimiento de gas natural desde Argentina obligó a nuestro país a recurrir al petróleo diesel para generación eléctrica, lo que implicó costos mayores entre tres a cuatro veces a los esperados de no haberse interrumpido el suministro de gas (Bitrán y González, 2010: 11). Por último, en el año 2008 nuestra economía sintió los efectos de la última gran crisis financiera, la que condujo a una baja en el crecimiento real del PIB que lo situó en $3,7 \%$ en 2008 y en $1,5 \%$ en 2009.

El saldo de estas crisis ha sido la caída constante de la productividad del país, en especial desde 1998 (ver gráfico 2). Durante la última década, el aporte de la formación de capital al crecimiento del PIB fue equivalente al de los años 1986-1997, pero las contribuciones del trabajo y la productividad total de los factores (PTF) cayeron (ver gráfico 3). Según la OCDE, la desaceleración del factor laboral explica cerca de un tercio de la reducción del crecimiento del PIB de la década pasada, y el visible estancamiento de la PTF, los dos tercios restantes (OCDE, 2011: 54). En este sentido Bitrán y González (2010:13) señalan que para haber mantenido las tasas de productividad anteriores a 1998, el país requería embarcarse en un proceso de reformas estructurales con el objetivo de aumentar la diversificación y sofisticación productivas, pasando de ser un exportador de recursos naturales a ser un exportador de productos de mayor valor agregado. Sin embargo, y contrariamente a la tendencia manifestada por otros países, Chile no adoptó estás políticas de innovación y su productividad disminuyó considerablemente.

3 En el caso del sector manufacturero chileno, el impacto del shock energético, dependiendo del sector, explica entre el 20\% y el 60\% de la caída de productividad (PTF) entre el periodo 92-99 y 2000-2005 (Bitrán y González, 2010: 12). 


\section{Gráfico 3}

Contribución de los tres factores al crecimiento económico 1986-2009

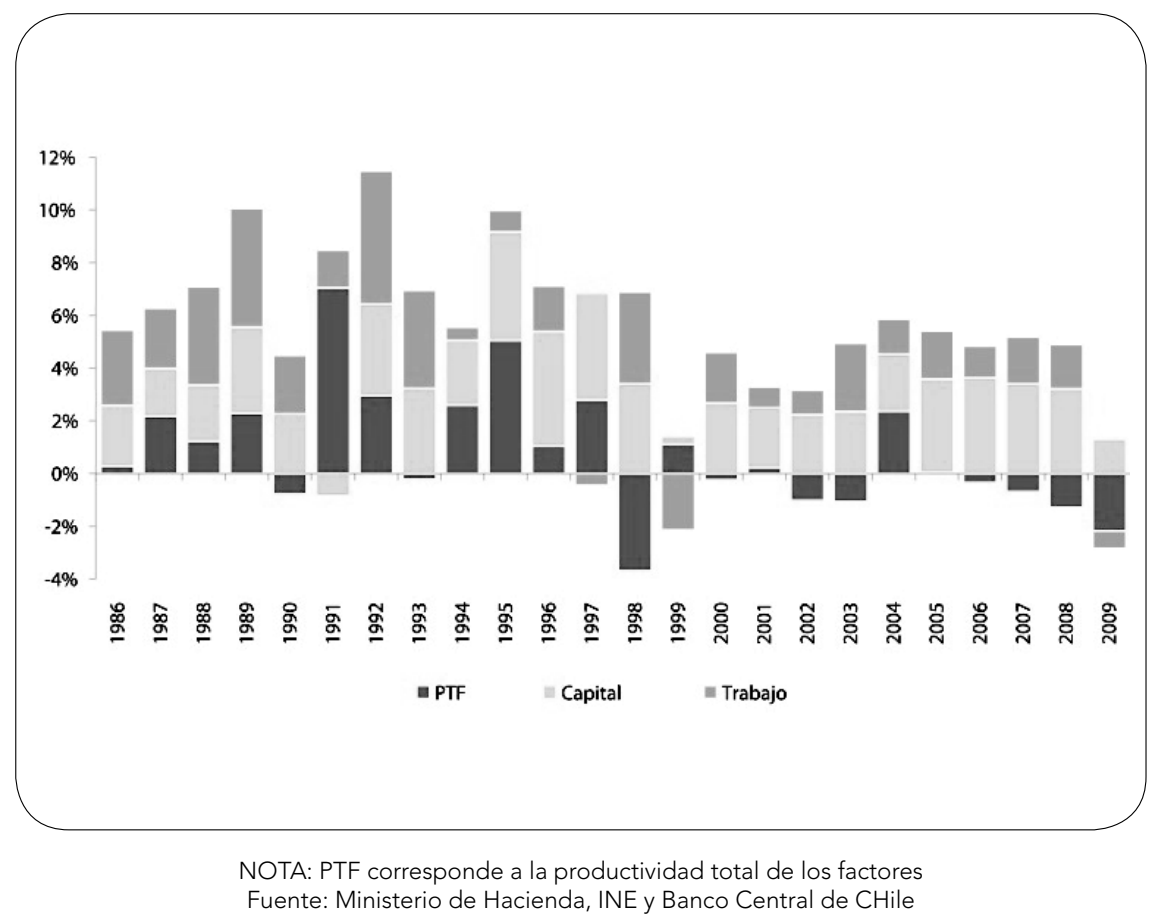

Las cifras sobre emprendimiento en la anterior década tampoco han sido auspiciosas. El ranking Doing Business del año 2007 señala a Chile en el lugar 39 respecto a las facilidades para iniciar una empresa y en el lugar 98 respecto a las facilidades para la quiebra. Así, en nuestro país toma en promedio 27 días comenzar una empresa, comparado con sólo 14,9 días promedio en la OCDE; y en promedio 3,2 años terminar una empresa, comparado con sólo 1,3 años para el caso de la OCDE (Bergoeing, 2007: 8).

Respecto a la innovación debemos decir, en primer lugar, que Chile tiene un bajísimo nivel de inversión en I\&D relativo a su PIB per cápita. Nuestra inversión en I\&D se ha mantenido prácticamente constante en un $0,7 \%$ del PIB en la década de 2000, manifestándose un continuo aumento de la cuota proveniente del sector público y una disminución de la proveniente del sector privado. Entre las principales consecuencias del poco desarrollo de I\&D del país podemos nombrar la baja calidad e insuficiente dotación de recursos humanos calificados, el mantenimiento de un bajo nivel de creación e incorporación de conocimiento a los procesos productivos 
de las empresas y la no incorporación de empresas de mayor productividad a la economía nacional. Según las conclusiones de un estudio del año 2010, a partir de los datos de la Encuesta de Innovación, de un universo encuestado de 4.000 empresas, el gasto en innovación como porcentaje del PIB habría disminuido de 1,6\% en 2003-2004 a un 1,2\% el 2005-2006 (Bitrán y González, 2010:18).

No obstante los efectos negativos de la última crisis, Chile demostró estar preparado para enfrentar la situación. Mediante un potente estímulo fiscal financiado con los excedentes del cobre y una sólida política de flexibilización monetaria, logró impulsar una recuperación temprana de la economía desde el último trimestre de 2009. Sin embargo, el terremoto de febrero de 2010 volvió a impactarnos negativamente, y consecuentemente tuvimos un crecimiento negativo de 1,5\% en el primer trimestre de 2010. Esto último no fue impedimento para que el país cerrase el 2010 con un crecimiento económico anual de 5,2\%. Para el año 2011, el Banco Central prevé un crecimiento de la economía dentro del rango entre 5,5\% a 6,5\% (Ministerio de Hacienda, 2010).

\section{Compromisos explicitados por el gobierno de Sebastián Piñera}

El Ministerio de Hacienda en su "carta de navegación" publicada en octubre de 2010 esquematiza los principales objetivos estratégicos del gobierno en el ámbito del crecimiento económico en la siguiente tabla:

\section{Cuadro 1}

Objetivos Estratégicos para el período 2010-2013

\begin{tabular}{|c|c|}
\hline \multirow{3}{*}{ Crecimiento } & $\begin{array}{l}\text { Alcanzar un crecimiento promedio del PIB de } 6,0 \% \text { en el período } \\
2010-2013\end{array}$ \\
\hline & $\begin{array}{l}\text { Aumentar la inversión, desde el 21,4\% actual, hasta un } 28 \% \text { del PIB al año } \\
2014\end{array}$ \\
\hline & $\begin{array}{l}\text { Aumentar la inversión en I+D, desde el } 0,4 \% \text { actual, hasta un } 0,6 \% \text { del PIB } \\
\text { al } 2013 \text { y } 0,8 \% \text { al } 2018\end{array}$ \\
\hline \multirow{3}{*}{ Empleo } & Crear 100.000 nuevos emprendimientos en el periodo 20102013 \\
\hline & $\begin{array}{l}\text { Crear } 200 \text { mil nuevos empleos promedio al año, durante el período } \\
2010-2014\end{array}$ \\
\hline & Capacitar a 5 millones de trabajadores en el periodo 2010-2013 \\
\hline
\end{tabular}

Fuernte: Ministerio de Hacienda, 2010 
Es tal la diversidad de políticas públicas relacionadas a crecimiento que actualmente está llevando a cabo el gobierno, que no resulta factible -dada la extensión de este trabajo- hacer un seguimiento completo de todas ellas. Por esta razón hemos preferido centrar nuestra investigación en la implementación de una política pública en particular: el sistema de análisis de impacto regulatorio ex ante que contiene el Estatuto Pyme.

\section{El sistema de análisis de impacto regulatorio ex ante que establece el "estatuto pyme"}

\section{a. El Estatuto Pyme}

El 3 de febrero de 2010 se publicó la Ley 20.416, que fija normas especiales para las empresas de menor tamaño (en adelante, EMT). El objetivo de esta ley es adecuar la carga regulatoria de estas empresas, las “pymes", de modo tal que la regulación tenga en cuenta sus características y no les resulte asfixiante.

Lo novedad de la ley es que incorpora por primera vez a nuestro ordenamiento un sistema general de análisis de impacto regulatorio, al establecer la obligación de todos los organismos públicos que dicten o modifiquen normas jurídicas generales que afecten a EMT, de realizar una estimación simple del impacto social y económico que la nueva regulación generará en las EMT. Previamente, se había establecido un mecanismo de análisis costo-beneficio en la Ley № 19.300 (v.g. planes de prevención y descontaminación ambiental) y en la Ley $N^{\circ} 19.966$ (garantías explícitas en salud).

\section{b. Aspectos teóricos del Análisis de Impacto Regulatorio (RIA)}

El Análisis de Impacto Regulatorio (regulatory impact assessment, RIA) es una herramienta de policy de aplicación sistemática que tiene por objetivo examinar los beneficios, costos y efectos esperados de la regulación nueva (RIA ex ante) o existente (RIA ex post), con el fin de ayudar a los tomadores de decisiones a optimizar la implementación de sus políticas públicas ${ }^{4}$. Nosotros nos centraremos en el RIA ex ante, es decir, en aquel que se sitúa temporalmente en las etapas iníciales del diseño de políticas públicas. Cuando se lleva a cabo en las primeras etapas del ciclo de diseño regulatorio el RIA ha demostrado ser una herramienta efectiva para mejorar la toma de decisiones estatales (OCDE, 2008: 15).

$4 \quad$ El Cabinet Office de Inglaterra (2003) define RIA como:”[A] tool which informs policy decisions. It is an assessment of the impact of policy options in terms of the costs, benefits and risks of a proposal" 
Los pasos que un RIA generalmente incluye son:

a. Definición del contexto de las políticas públicas propuestas y sus objetivos.

b. Identificación de todas las posibles opciones reglamentarias y no reglamentarias que permitirán alcanzar el objetivo de política pública que se persigue.

c. Identificación y cuantificación de los impactos de las opciones consideradas, incluyendo costos, beneficios y efectos distributivos.

d. El desarrollo de estrategias de observancia y cumplimiento para cada opción, incluyendo una evaluación de su eficacia y eficiencia.

e. El desarrollo de mecanismos de monitoreo para evaluar el éxito de la propuesta.

f. La implementación de consultas públicas que proporcionen a todos los interesados la oportunidad para participar en el proceso regulatorio.

\section{c. El Sistema de Impacto Regulatorio que establece el Estatuto Pyme}

El artículo quinto de la Ley 20.416 (“Estatuto Pyme”), vigente desde el $1^{\circ}$ de septiembre del 2010, establece el procedimiento para la dictación de Reglamentos y Normas de Carácter General que afecten a EMT. Dispone que aquellos organismos sujetos al procedimiento deben publicar los antecedentes preparatorios necesarios para la formulación de dichos reglamentos y normas, y que estos antecedentes deben contener una estimación del impacto social y económico que la nueva regulación tendrá sobre las EMT. Lo anterior se traduce en las siguientes obligaciones legales:

a. Obligación para los órganos de la Administración del Estado que dicten regulaciones de carácter general con efectos sobre EMT, de enviar las propuestas al Ministerio de Economía, Fomento y Turismo, con al menos 15 días de anticipación, salvo regulaciones urgentes.

b. Obligación para los órganos de la Administración del Estado que dicten regulaciones de carácter general con efectos sobre EMT, de publicar - por regla general, con al menos 15 días de anticipación a la dictación de la norma y en la página de Gobierno Transparente- los antecedentes preparatorios que sustentan dicha normativa. Esta publicación se realiza mediante el llenado del "Formulario de Estimación de Impacto Regulatorio de Empresas de Menor Tamaño”, el cual

Con el objeto de uniformar la publicación de los antecedentes preparatorios el Ministerio de Economía puso en su sitio web a disposición de los órganos estatales el formulario estándar y un manual de instrucciones para completarlo. 
contiene una serie de preguntas que deben responder los órganos estatales con información sobre las regulaciones que proponen. Son estos formularios los que posteriormente son publicados en el sitio web de Gobierno Transparente.

Cabe notar que el incumplimiento u omisión de las obligaciones mencionadas no afecta en caso alguno la validez del acto regulatorio propuesto.

\section{d. Análisis de la implementación del Sistema de Impacto Regulatorio que establece el Estatuto Pyme}

Para conocer la implementación del sistema de RIA que establece el Estatuto Pyme hemos procedido a analizar los Formularios de Estimación de Impacto Regulatorio respondidos por los órganos estatales que dependen de aquellos ministerios que, en nuestra opinión, poseen mayor incidencia regulatoria sobre las EMT. Dichos ministerios son: Economía, Hacienda, Salud, Transportes, Trabajo, Educación, Obras Públicas, Minería y Energía. La muestra analizada se compone de un total de 97 formularios, todos ellos publicados en el sitio web de Gobierno Transparente.

\section{Gráfico 4}

Número de formularios encontrados por Ministerio

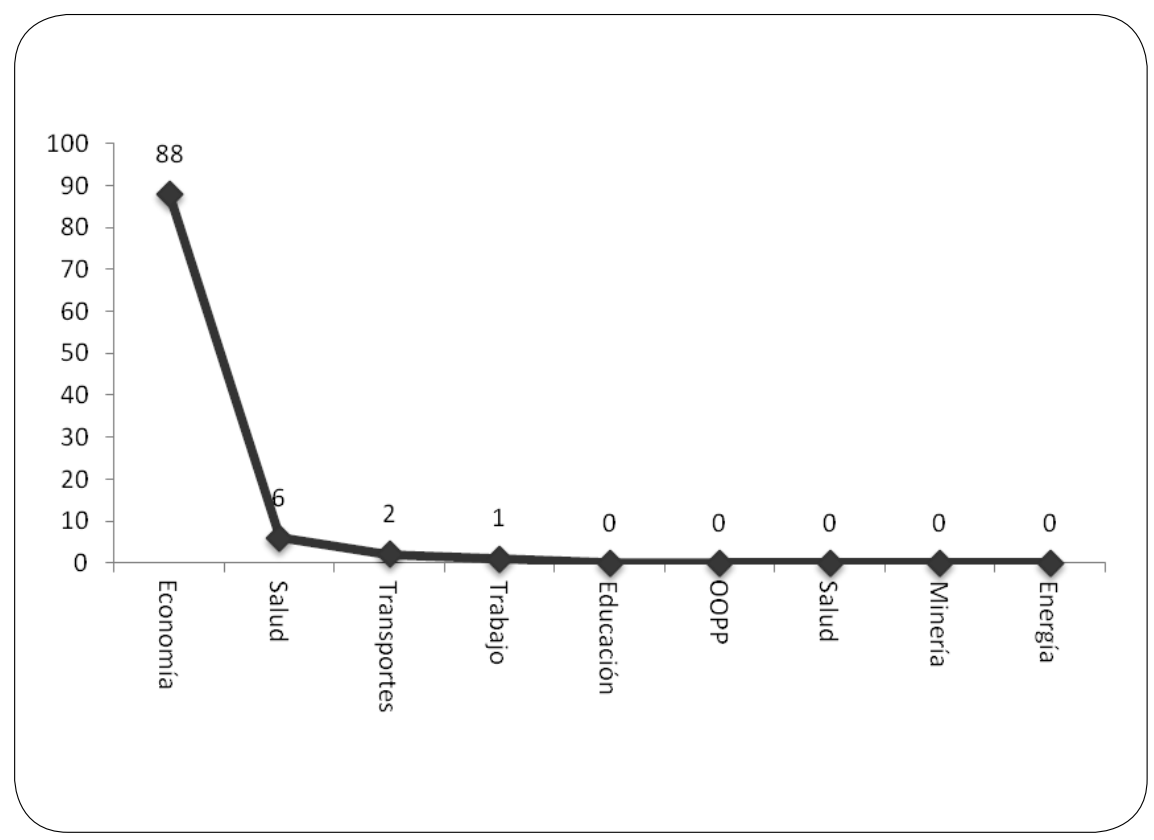

Fuente: Elaboración propia a partir de formularios obtenidos de la sección Antecedentes preparatorios de normas que afecten a EMT Ley $N^{\circ} 20.416$, del sitio web www.gobiernotransparentechile.cl 
La primera sorpresa fue constatar que 88 de los 97 formularios que encontramos provienen del Ministerio de Economía (ver gráfico 4). Lo anterior es relevante por cuanto podría ser interpretado como que la única secretaría de Estado que efectivamente ha cumplido con el artículo $5^{\circ}$ de la Ley 20.416 ha sido precisamente el Ministerio encargado de la implementación de dicho estatuto legal.

Mientras, el resto de la Administración del Estado a la cual está dirigida la obligación del artículo $5^{\circ}$ de la Ley 20.416 no parece haber dedicado ni tiempo ni recursos suficientes al cumplimiento de la ley. Resulta dudoso que ministerios como el de Salud y Transportes no hayan dictado regulaciones con impacto sobre las EMT desde septiembre de 2010 a la fecha.

\section{Gráfico 5}

Actividad económica a la que se le aplica la norma

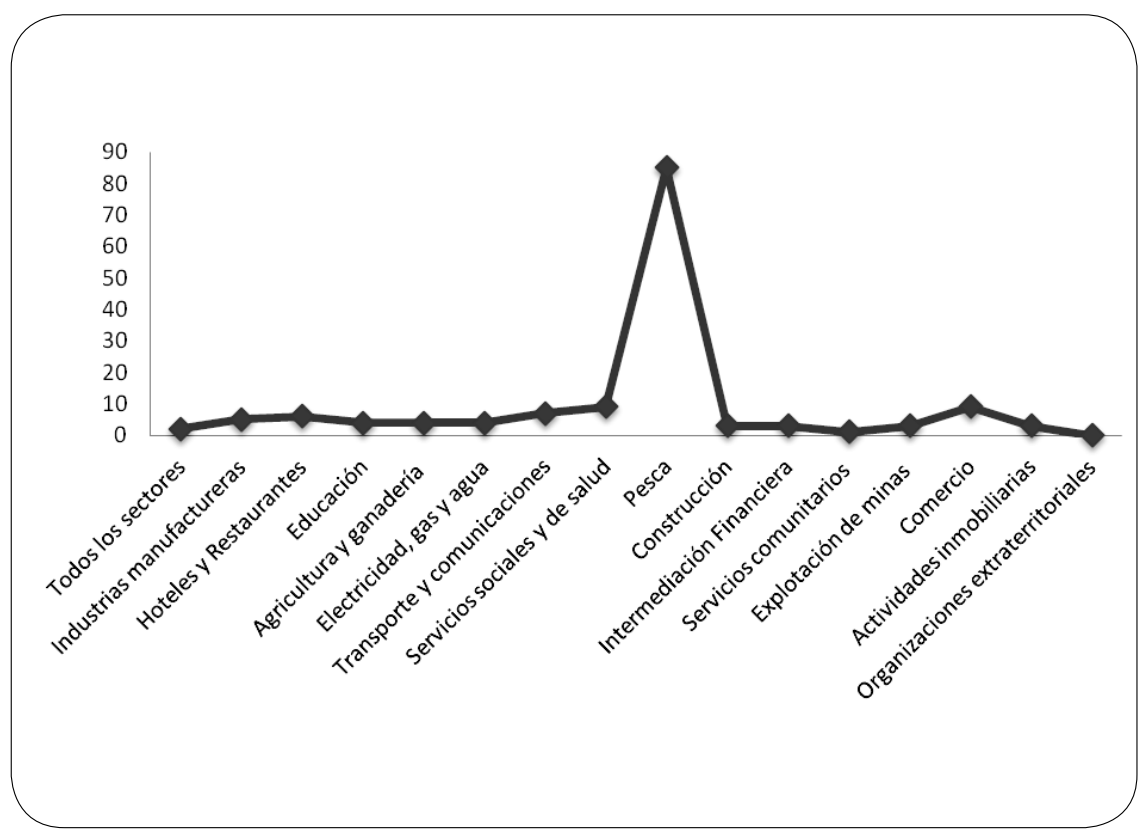

Fuente: Elaboración propia a partir de formularios obtenidos de la sección Antecedentes preparatorios de normas que afecten a EMT Ley $N^{\circ} 20.416$, del sitio web www.gobiernotransparentechile.cl

Una segunda sorpresa encontramos al analizar las actividades económicas que son afectadas por las propuestas regulatorias. Frente a la pregunta sobre las actividades económicas a las que se aplica la norma, existen 85 respuestas que señalan a la pesca como la actividad afectada, mientras que en segundo lugar se sitúan los servicios 
sociales y de salud y el comercio, con sólo nueve respuestas (tal como se expresa en el gráfico 5).

Respecto a otras respuestas que encontramos, queda la impresión de que la implementación de sistema de RIA del Estatuto Pyme tiene un arduo camino por delante. Esto porque parece existir cierta inercia en los servicios públicos a la hora de tomar sus decisiones regulatorias -inercia que resta efectividad a las políticas públicas pro EMT-y que consiste en que los servicios públicos, en especial los menos sofisticados, no acostumbran considerar diversas alternativas regulatorias a la hora de fijar sus normativas en general y las sus regulaciones para EMT en particular. Lo anterior se refleja en el hecho de que frente a la pregunta contenida en el formulario de si se consideraron alternativas regulatorias o no regulatorias que luego hayan sido descartadas en favor de la propuesta actual, sólo en un $8 \%$ de los formularios se contesta afirmativamente. Contrasta lo anterior con las respuestas que encontramos a la pregunta de si fueron consultados los contenidos de la propuesta regulatoria con otras entidades relevantes. En este último caso, en un $89 \%$ de los formularios se señala que efectivamente se habrían realizado dichas consultas. Respecto al tipo de entidades a las que se dirigieron estas consultas, $27 \%$ de ellas resultaron ser dirigidas a organismos del Estado, $22 \%$ a entidades gremiales, $16 \%$ a centros de estudio y el 35\% restante a entidades de otro tipo (ver Gráfico 6).

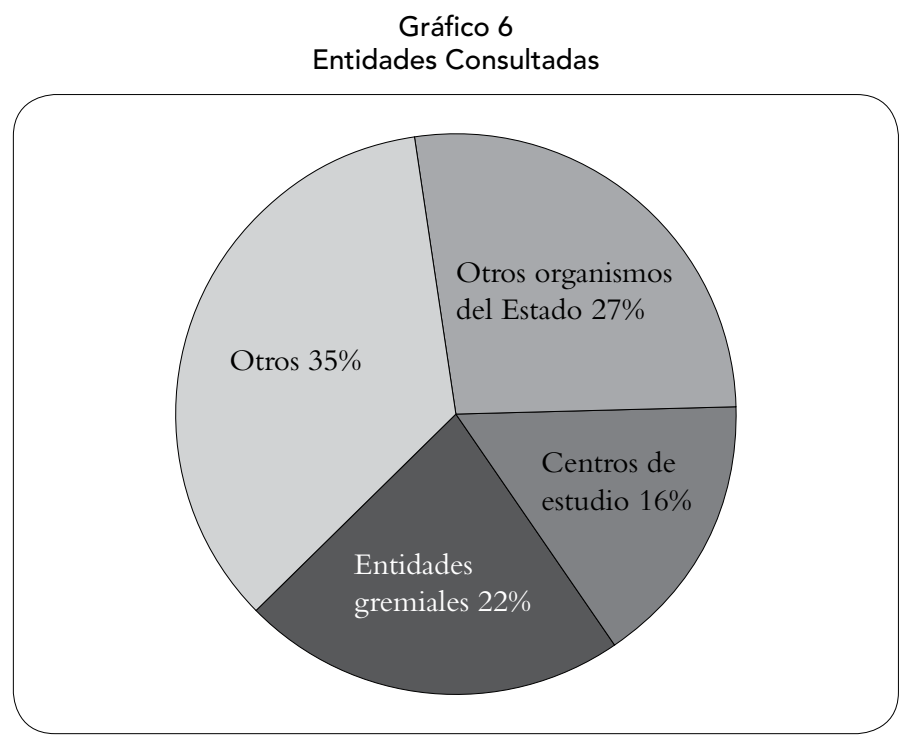

Fuente: Elaboración propia a partir de formularios obtenidos de la sección Antecedentes preparatorios de normas que afecten a EMT Ley $N^{\circ} 20.416$, del sitio web www.gobiernotransparentechile.cl 
Frente a la pregunta del formulario a si la propuesta considera una diferenciación y/o exención según tamaño de la empresa a la que se le aplica la norma, un 89\% de los formularios muestra que no se considera diferenciación ni exención alguna. Este resultado parece contrastar negativamente con el objetivo perseguido por el Gobierno respecto de las EMT, es decir, en adecuarles la carga regulatoria de modo tal que esta no les sea asfixiante.

Al preguntar si la propuesta genera beneficios para las EMT, en 59\% de los formularios se responde afirmativamente. Respecto de los beneficios específicos que se espera generen las propuestas, en primer lugar está la mejora en el acceso a mercados (27\%), seguido por la mejora en condiciones laborales (18\%) y la mejora en el acceso al financiamiento (15\%). En contraste, la eliminación y abaratamiento de trámites constituyen una proporción muy baja de los beneficios que se espera generen las propuestas regulatorias analizadas para las EMT (5\% y 3\%, respectivamente). No obstante las respuestas anteriores, al preguntar si se tiene una estimación cuantitativa de los beneficios que generaría la propuesta para las EMT se constata que en un $82 \%$ de los casos la respuesta es negativa.

\section{Conclusiones y Recomendaciones}

La implementación del sistema de análisis regulatorio que incorpora el Estatuto Pyme es una novedosa iniciativa con potencial de aumentar exponencialmente la eficiencia de las políticas públicas que afecten empresas de menor tamaño. No obstante, una mirada rápida a los resultados que se observan en los Formularios de Antecedentes Preparatorios permite concluir que, en los ocho meses que lleva vigente la iniciativa, esta no ha encontrado en la práctica el apoyo efectivo de la mayor parte de los Ministerios. Salvo por el Ministerio de Economía (y en especial, por la Subsecretaría de Pesca), las demás carteras prácticamente no han completado formularios de antecedentes preparatorios, siendo poco probable que durante los ocho últimos meses no hayan dictado regulaciones que afecten a empresas de menor tamaño.

Respecto a las propuestas regulatorias contenidas en los formularios, llaman negativamente la atención los siguientes puntos:

a. Los servicios públicos prácticamente no consideran alternativas a la hora de diseñar sus propuestas regulatorias. 
b. Las normas propuestas prácticamente no distinguen según el tamaño de la empresa a la cual se aplican.

c. Los servicios públicos prácticamente no realizan estimaciones cuantitativas de los beneficios que generan sus propuestas.

Respecto a la dificultad para introducir un sistema de RIA en el aparato estatal, creemos que ello se explica por tres causas principales: (i) el hecho de que los distintos servicios públicos no entiendan la utilidad de llevar a cabo un RIA, (ii) la falta de un sistema de incentivos (o sanciones) para que los servicios públicos cooperen con la implementación del sistema y (iii) la amplitud excesiva del sistema de RIA que consagra el Estatuto Pyme, considerando que es un plan piloto en nuestro país.

Respecto al hecho de que los distintos servicios públicos no entiendan la utilidad de llevar a cabo un RIA, Keyworth y Yarrow (2006) sostienen que existe una tendencia a ver al RIA sólo como un documento o un conjunto de documentos que se acompaña a una propuesta de regulación en una etapa inicial del proceso de toma de decisiones administrativas. Para revertir esta tendencia, el órgano que implementa el RIA (en este caso, el Ministerio de Economía) debe llevar a cabo campañas de información con el objeto de concientizar a los servicios públicos de que el RIA es un proceso de examen y evaluación de alternativas regulatorias que contribuye a mejorar la eficiencia de las políticas de la Administración. Una vez que esto se entiende claramente, será más fácil que los servicios públicos vean al RIA en una perspectiva de largo plazo y valoren su aporte al proceso de decisión política.

El éxito en la implementación de un sistema de RIA requiere de incentivos dirigidos a los servicios públicos, en especial cuando el incumplimiento de las obligaciones que configuran el sistema no genera sanción legal alguna.

El Gobierno debe ser realista acerca de la escasez de recursos financieros y humanos necesarios para la apropiada implementación de un RIA, en especial cuando esta constituye una iniciativa pionera en el país. En consideración a ello creemos que resulta excesivamente amplio un sistema que pretende que todos los ministerios $\mathrm{u}$ organismos que dicten o modifiquen normas jurídicas generales que afecten a empresas de menor tamaño, con excepción de las ordenanzas municipales y de los dictámenes que puedan emitir los órganos de la Administración del Estado, deban realizar una estimación simple del impacto social y económico que la nueva regulación generará en dichas empresas. Un sistema de tal envergadura necesita comen- 
zar con una etapa de implementación en formato piloto, para después ser escalado, dependiendo de su éxito y de las lecciones que se aprendan en el camino.

Creemos que un sistema realista de RIA para el ámbito de las empresas de menor tamaño de nuestro país debiese estar dividido en dos etapas: un RIA previo y simple, y un RIA posterior y detallado. Este enfoque de dos etapas comienza con un RIA previo y de bajo costo de implementación, cuyo objetivo es identificar las regulaciones que deben ser objeto de un RIA posterior más detallado. Esta identificación debe realizarse mediante "filtros" que se aplicarán a las propuestas de regulaciones y que determinarán -sobre la base de umbrales o criterios predefinidos- aquellos casos donde se justifique la realización de un RIA completo. Estos umbrales pueden estar expresados en términos monetarios, si es que consisten en determinados niveles de costos y/o beneficios que potencialmente pueda generar la regulación en análisis, o en aspectos como, por ejemplo, el impacto potencial de la regulación sobre la competencia, empleo, productividad o innovación.

La utilidad de un sistema de RIA depende directamente de la calidad de la información utilizada para evaluar el impacto de las propuestas regulatorias. Una estrategia pobre de recopilación de información puede significar que los datos necesarios para llevar a cabo un buen análisis serán insuficientes, y que por ende sus resultados pueden ser contraproducentes. Es por ello que para llevar a cabo eficazmente un RIA, el Gobierno debe establecer directrices precisas y detalladas que los ministerios deben cumplir al hacer los análisis de sus propuestas regulatorias. Esto implica, también, que los responsables políticos necesitan ganar habilidades, pensar en términos cuantitativos y familiarizarse con la recopilación de datos y el análisis costobeneficio. Una implementación óptima de un sistema de RIA requiere la flexibilidad suficiente para que la recolección de información se adapte a las características y objetivos propios de las regulaciones propuestas.

No obstante las mejoras que necesita el sistema, afirmamos que este cumple un adecuado rol de transparencia en la formulación de políticas públicas, además de introducir el concepto de "empresa de menor tamaño" como un criterio a tomar en cuenta por los órganos estatales al emitir sus regulaciones. Debe señalarse que las dificultades e inconvenientes que se observan en la implementación del sistema de RIA ex ante del Estatuto Pyme, a más de ocho meses de su vigencia, son análogas a las dificultades de implementación que se han presentado en otros países OCDE en situaciones de implementación de sistemas similares. 


\section{Referencias bibliográficas}

Banco Central (2011). Informe de Política Monetaria (marzo). Santiago: Banco Central de Chile.

Bergoeing, Raphael (2007). Morir no es malo: el éxito nace del fracaso. Seminario Reformas Micro. Santiago: Centro de Estudios Públicos.

Bitrán, Eduardo y González, Cristián (2010). Documento de referencia: Productividad Total de Factores, Crecimiento e Innovación. Santiago: Consejo Nacional de Innovación para la Competitividad.

Cabinet Office (2003). Better Policy Making: A guide to Regulatory Impact Assessment. Londres: Cabinet Office Regulatory Impact Unit.

Cerda, Rodrigo y Larraín, Felipe (2005). "Inversión Privada e Impuestos Corporativos: Evidencia para Chile”. Cuadernos de Economía,Vol. 42, N 126, pp. 257-281.

Ffrench-Davis, Ricardo (2002). "El impacto de las exportaciones sobre el crecimiento de Chile”. Revista de la CEPAL N 76, pp.143-160.

Keyworth, Tim \&Yarrow, George (2006). Revising the Regulatory Impact Assessment: Response to the BRE's Consultation. Londres: Regulatory Policy Institute.

Ministerio de Economía, Fomento y Turismo (2011). Minuta Ley 20.494 del 27 de enero que facilita la constitución y funcionamiento de nuevas empresas. Santiago: Gobierno de Chile.

Ministerio de Hacienda (2010). Chile, País Desarrollado. Santiago: Gobierno de Chile.

Ministerio de Hacienda (2010). Estado de la Hacienda Pública. Santiago: Gobierno de Chile.

OCDE (2008). Building an Institutional Framework for Regulatory Impact Analysis (RIA). OECD Publishing.

OCDE (2011). Mejores Políticas para el Desarrollo: Perspectivas OCDE sobre Chile. OECD Publishing.

Piñera Echeñique, Sebastián (2010). "Del Chile del bicentenario al país de las oportunidades”. Mensaje a la Nación 21 mayo.Valparaíso: Gobierno de Chile. 